\title{
INFORMATICS SYSTEM FOR SUPERVISION AND CONTROL OF PROCESSES WITH MOBILE DEVICES
}

\author{
Elizabeth M. Astorga GonzÁlez*, Roberto Acosta González*, Pedro Milá Ortiz*, Pedro \\ M. G. DEL Foyo ${ }^{\dagger}$, José Reinaldo Silva \\ * Informatics Engineering Department, Universidad de Oriente \\ Santiago de Cuba, Cuba \\ ${ }^{\dagger}$ Mechanical Engineering Department, Federal University of Pernambuco \\ Recife, PE, Brazil \\ $\ddagger$ Mechanical Engineering and Mecatronic Systems Department, University of Sao Paulo \\ São Paulo, SP, Brazil
}

Emails: elizabeth.astorga@inf.fie.uo.edu.cu, raglz@fie.uo.edu.cu, pmila@fie.uo.edu.cu, pgdelfoyo@yahoo.es, reinaldo@usp.br

\begin{abstract}
Control and Automation has experienced a tendency to use wireless devices for supervision and control of different process, which also improved with the development of mobile technology. This paper shows how to develop an informatics system for supervising and controlling processes using mobile devices, implementing an architecture that uses wireless connection ( $\mathrm{Wi}-\mathrm{Fi}$ ) and a fieldbus to establish communication with the processes. The mobile devices are based on Android which runs an application that establish communication with a computer running a server that listen all requests and sends, by RS232, the data sent by the mobile device through the fieldbus to the process. For communication between different elements of the system a RS232 protocol was used. This protocol is a modification of the original RS232 adjusted to the architecture of the system.
\end{abstract}

Keywords_ Supervision and control, Mobile device

\section{Introduction}

Since the arrival of the new generation of mobile devices and the development of wireless communications, mobile devices became attractive as a way to retrieve all kind of information from access to social networks up to applications that handle and display specific contents. As a consequence, industry related applications customized their applications to handle data by mobile phones.

In current technological advances, it is common the access the operating state of processes by a network, starting from web applications and going to web services that can be used in diverse platforms. Also, using of mobile devices to perform tasks such as monitoring is very appealing, since data can be consulted at any time no matter were the mobile device is located. Despite not having the same processing power than conventional computers such devices rely on modern operating systems that allow them to perform the required tasks using available resources very efficiently.

\section{Current types of supervision and process control}

Today, the industry has automated more complex production processes in which coexist a wide variety of items: computers, pneumatic drives, robots, and so on. However, in order to fulfill the current demands in manufacturing such systems must be flexible enough to provide rapid answers to a fre- quently changing market. Off course, the improvement in productions systems flexibility would also increase the complexity of the control system necessary to govern it. This brought to the development of "smart" control systems, based on concepts such as decentralization, autonomy, monitoring, cooperation and collaboration. The increased complexity leads to a demand for well trained staff for maintenance and development of those systems.

Also, as a result of these frequent changes in production processes, the reliability, suitability and standardization of these changes became a main issue in the implementation of this new technology (Chacon et al., 2001).

There is an urgent need to facilitate the monitoring and control of processes. Because of that different types of architecture have been adopted to facilitate control by using innovative technologies such as mobile devices.

In the following we present the most common forms of control used today

\subsection{SCADA (Supervisory Control And Data Ac- quisition)}

SCADA systems are software applications designed to control and monitor remote processes. They are based on data acquisition from remote processes. This is a software application specially designed to run on computers to control production, providing communication with plant devices (standalone controllers, PLCs, etc.) and control- 
ling the process automatically from a computer. It also sends information generated in the productive process to different users, as other supervisors within the company, so that it allows the participation of other production areas such as: quality control, monitoring, maintenance, and others (Ramirez and Chingate, 2010).

\subsection{Monitoring and Controlling via web}

Due to the development of Internet the chances of running a remote process has been made possible, leading to the use of websites and web services to create applications that are able to communicate with specific processes by receiving alarms, viewing the status of each process, or controlling the performance of actions.

One of the most significant advantages these Web applications is their installation and distribution, were all strength is applied configuring the server side of the components and not in the client side. This is a breakthrough in terms of control systems mentioned here because it allow that the control and supervision does not necessarily have to be done in the plant level but could be moved anywhere in the factory or even to the outside.

\subsection{Supervision and remote control using mobile devices}

Mobile devices are actually used in industry and supervisory panel for control and monitoring of processes, variables and/or external devices as well as measuring physical variables and sending that information to a control center. Many control systems today use this technology to expand the monitoring of their systems.

Some of the technologies used to implement this solution are: SMS, wireless transmission, WiFi and Bluetooth. These solutions constitute a new way to monitoring systems primarily through devices like micro controllers, PLCs, among others. Systems incorporating mobile technology have all intermediate architecture to perform traditional control, such as SCADA systems, using OPC for obtaining process data, or the use of assistive devices such as GSM modems, connected directly to the process controller, PLC or micro controller.

This paper proposes the creation of an architecture for mobile devices that allows getting data and preforming action directly on the process without using any devices connected to the process controller like GSM modems, but only by using a wireless connection via $\mathrm{Wi}-\mathrm{Fi}$ and serial communication.

\section{Supervising and controlling systems with mobile devices}

There are several systems in the world that perform control and supervision, offering different types of architecture, that include the use of mobile devices.

\subsection{Multiplatform industrial system for supervis- ing and controlling}

This system provides an architecture platform from which you can obtain process data through OPC server (OLE for Process Control) and was used in (Coral et al., 2008). Figure 1 shows the system architecture.

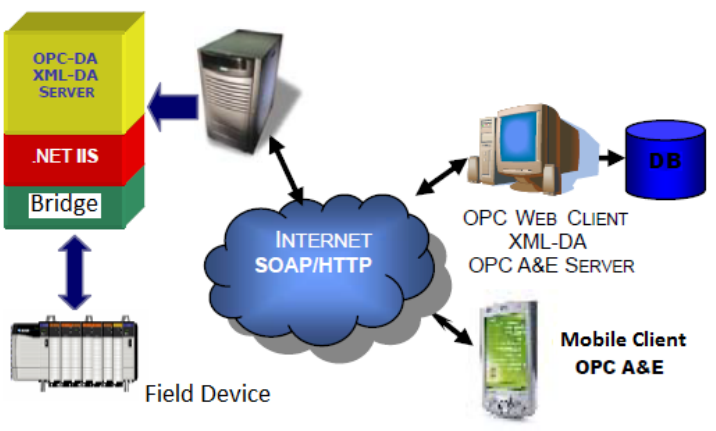

Figure 1: Architecture of the Multiplatform industrial system for supervising and controlling

This architecture has as client and two types of users: a mobile device that can access a server OPC, Alarms and Events over the Internet, via WAP, and DAXML, an OPC client that accesses data in the Internet, through SOAP and HTTP, but is also an OPC Alarm and Events server with historical data access using JDBC. The mobile client within the system does not have many possibilities since it is only limited to act as a supervisor of alarms and events, and has no history record of his own or stores data. Also, it does not have the ability to act in the process.

\subsection{Control and Monitoring System based re- mote mobile GSM}

This approach was used in (Boter et al., 2005). System monitors and control isolate plants from any remote location using a mobile phone using a GSM modem connected to a PLC. The system is capable of controlling the plant by sending and receiving short text messages, particularly SMS (Short Message Service), which may be redirected to different phone numbers, according to established protocols in the application. These messages predetermined or incorporating state variables can be considered like alarms if the PLC generates them, or answers to requests for informa- 
tion from the operator. In the Figure 2 is shown how the system is conceived.

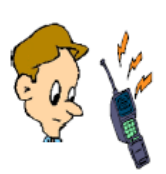

USER
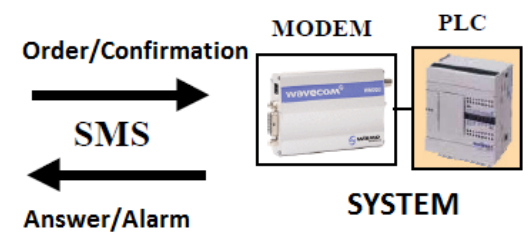

SYSTEM
Figure 2: Architecture of a controlling an monitoring system using GSM technology

This system although meets requirements for monitoring and control via mobile devices processed independently and directly connected to it, can be used only when it is a PLC control device or automata. Besides, the system does not provide access to historical data and there is no way of configuring the system to give different kind of access to distinct users like in other control and supervision system.

\section{Proposed system architecture}

The proposed system for monitoring and controlling processes form mobile devices has an architecture that enables ghatering data from the process and send operation to the process without any SCADA system.

The system is based on the use of wireless technology via $\mathrm{Wi}-\mathrm{Fi}$ to establish communication with the mobile device, and uses a fieldbus for adding new control elements to get the data using BISYNC* as communication protocol between the different elements (See Figure 3).

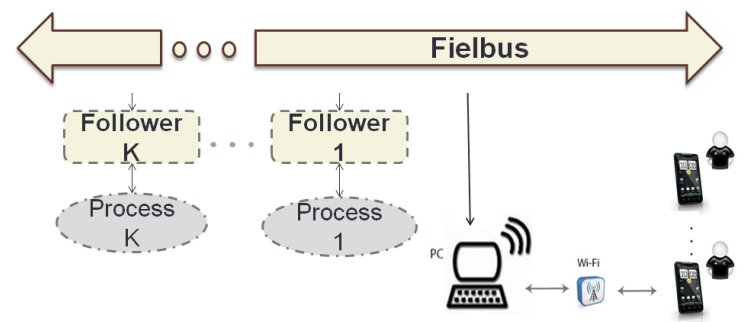

Figure 3: Architecture of the proposed system

The system itself consists of mobile devices with a mobile application that work with sockets, using TCP/IP via $\mathrm{Wi}-\mathrm{Fi}$, and sends data to a server that listen to the requests. The server takes the information and turns it into an array of bytes to be sent via RS232 using the communication protocol BISYNC*. The information is received by a process that performs an action: even send data or perform a controlling action, or both.

In the following we describe the control elements to which an action are sent. Each element has an address that allows them to get only the data addressed to them.

In the case of lost of communication, the control is not affected because the control algorithm is embedded in the controller not in the mobile device or PC.

\section{$4.1 B I S Y N C^{*}$}

BISYNC stands by Binary Synchronous Communication, a protocol for data communication developed by IBM (International Business Machines) in 1967. It is a character-oriented protocol to facilitate asynchronous transmissions (Hall, 2006).

For convenience it was decided to amend the protocol to suit the needs of the system, kept the name, and just add the character "*" to differentiate it from the original. This protocol is used to send data to all system components. It is formed by two types of frames, the command and data (See Figure 4.
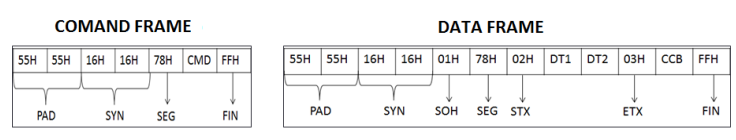

Figure 4: Data frame from protocol BYSINC*

In the command frame, the CMD field coincides with one of the proposed protocol commands ACK, ENQ, NAK or EOT (Hall, 2006); data frame DT1 and DT2 are the fields through which a combination of actions are set to do the process, and SEG is the field used to identify which component is supposed to acquire it.

\subsection{System access levels}

For security features the system has three levels of access: operator, supervisor and administrator. The system operator can perform actions of automatic control, edit the references for the system variables and perform on/off actions; the supervisor only observes the state of the process while the administrator can perform automatic or manual control, and also can get a list of users connected to the system.

The access level are asigned by the administrator of the system. Only one user with administrator or operator level can control the systen. In case more than one user with the same acces level is connected to the systen only accepts the first one connected as controler

If the controler disconnects to the system, the control is given to the first user on the list with administrator or operator level; in case of nonexistence of any user with these level the system will work only with accion given by the control algorithm embedded in the controller. 


\subsection{The Server}

The process server is responsible for obtaining data via RS232, send it to the process and also to the corresponding mobile device, and also sets the working mode of users, and monitors the connected users with the system, storing their data and information about the connection status.

The server has a database capable of storing users identification with their permission access to the system and the data sent by the process, alarms and the variables status. The implementation of this element of the system was performed using the Java programming language, programed in the IDE NetBeans 7.0.

\section{A Case Study}

To show the system implementation a case study was chosen for test, in this case the prototype is based in an electric oven.

In the oven, heat is obtained by an electrical resistor, which is powered by thyristors connected in series to them. The oven also has devices to simulate sources of noise or disturbances in the process variable, a soldering iron to simulate the temperature rise and a fan to insufflate air into the oven and decrease the temperature. Both elements can be operated remotely or locally, usually manually. To measure the temperature a thermistor is used.

The control element for this process is the PIC 18F452 microcontroller that contains the control algorithm of the process and interprets the actions sent by the mobile device to be executed. This micro controller in the system architecture is one of the elements connected to the fieldbus.

\subsection{Data Frames for comunication}

Using the protocol BISYNC* different data frames were defined in order to ensure de comunication from/to the process. Table 1 shows the data frames for our case study.

\subsection{Mobile Application}

The mobile application was developed in Android and has a friendly user interface to interact with the process, offering the state of the process, temperature and buttons to operate the elements of disturbance; a graph of the temperature, the temperature history and alarms.

The interface are dependents on the access level for the user that enters the system; there are different user interfaces which matches the three levels of security: operator, administrator or supervisor.

To send data and command frames from the mobile application through sockets some classes

\begin{tabular}{|l|l|l|}
\hline DT1 & $\mathrm{DT} 2$ & Meaning \\
\hline $74 \mathrm{H}$ & $V T$ & $\begin{array}{l}\text { Temperature read by the } \\
\text { microcontroller }\end{array}$ \\
\hline $65 \mathrm{H}$ & $\mathrm{X}$ & $\begin{array}{l}\text { Operate the oven, where } \mathrm{X} \\
\text { can be } 69 \mathrm{H} \text { (on) or } 6 \mathrm{FH} \text { (off) }\end{array}$ \\
\hline $62 \mathrm{H}$ & $\mathrm{X}$ & $\begin{array}{l}\text { Operate the fan, can be } \\
\text { done } \mathrm{X} 69 \mathrm{H} \text { (on) or } 6 \mathrm{FH} \text { (off) }\end{array}$ \\
\hline $64 \mathrm{H}$ & $\mathrm{X}$ & $\begin{array}{l}\text { Apply the soldering iron, } \\
\text { where } \mathrm{X} \text { can be } 69 \mathrm{H} \text { (on) } \\
\text { or } 6 \mathrm{FH} \text { (off) }\end{array}$ \\
\hline $61 \mathrm{H}$ & $V R$ & $\begin{array}{l}\text { Reference temperature, } \\
V R \text { is the reference value }\end{array}$ \\
\hline $6 \mathrm{DH}$ & $\mathrm{X}$ & $\begin{array}{l}\text { Alarm, where } \mathrm{X} \text { can be } 66 \mathrm{H} \text { (off) } \\
63 \mathrm{H}(\geq 70 \text { and } \leq 85 \text { degrees) } \\
\text { and } 67 \mathrm{H} \text { ( } \geq 85 \text { degrees) }\end{array}$ \\
\hline $68 \mathrm{H}$ & $\mathrm{X}$ & $\begin{array}{l}\text { Mode, where } \mathrm{X} \text { can be } 79 \mathrm{H} \text { (aut } \\
\text { mode) or } 7 \mathrm{AH} \text { (manual mode) }\end{array}$ \\
\hline $6 \mathrm{BH}$ & $V P$ & $\begin{array}{l}\text { Power for the PWM, where } \\
V P \text { is the value that is assigned } \\
\text { directly to the PWM }\end{array}$ \\
\hline $6 \mathrm{AH}$ & $0 \mathrm{x} 00$ & \begin{tabular}{l} 
Reading the status of the process \\
\hline
\end{tabular} \\
\hline
\end{tabular}

Table 1: Combination of the DT1 and DT2 fields to send actions to the process

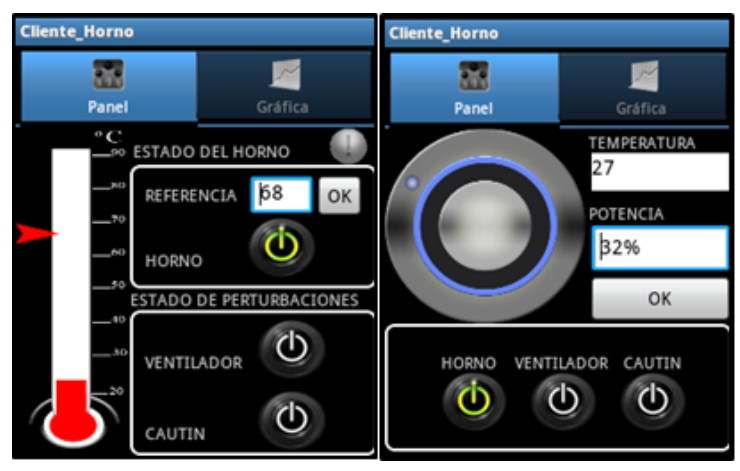

Figure 5: User interface in the mobile

were implemented to represent each of these frames and were sent via $\mathrm{Wi}-\mathrm{Fi}$ to the server.

The system ensures that in the case of disconnection of the user who is performing the control, another user can take control of the process, because the system is responsible for finding another user who is connected that has permissions to perform the controlling tasks. In the absence of another connected user the process continues working in automatic control mode independently by the microcontroller PIC 18F452.

Since the control algorithms are executed by the microcontroller PIC 18F452 there are no concern about communication failures impacting process control performance. The deterministic behavior of the control algorithm is guarantied despite the communication status. Some degradation can be expected since setpoint or even control strategies modifications can be done only when communication is active. 


\begin{tabular}{|c|c|c|}
\hline $\begin{array}{c}\text { Distance } \\
\text { (meters) }\end{array}$ & Coverage & $\begin{array}{c}\text { Response Time } \\
\text { (seconds) }\end{array}$ \\
\hline 5 & maximum & 2 \\
\hline 10 & maximum & 2 \\
\hline 30 & medium & 5 \\
\hline 40 & medium & 5 \\
\hline 54 & low & 7 \\
\hline
\end{tabular}

Table 2: Time of response of the system in different Wi-Fi covered

The mobile application in this case study was implemented on an Android smartphone but can be created for any other mobile operating system with the same design.

\section{Results}

The system was tested several times to test its performance in different situations. Mostly, the system performance with different Wi-Fi covered. The results are shown in Table 2 . The device used to perform these tests was a TP-LINK 54M Wireless Router.

The system offers simultaneous connection to different users, and it was tested with a maximun of 12 users conected at the same time; one in automatic control mode and the others in monitoring mode. The system did not present any problem of abrupt disconnection and all data of the process was sent to all users.

The control system performance (oven temperature) was never compromise under any configuration of users roles.

\section{Conclusion}

The informatic system ensures the control and monitoring of process using mobile devices independently, providing more versatility to process of control and monitoring.

The test of the system in several situations shows the a good performance and accuracy. The time of response of the system was satisfactory for purpose of the system, that is, settting a reference point, and action on/off over the element is not critical because the real control of the process will be made by the control device in an aceptable time interval.

The system arquitecture presents a new way for adding the mobile devices to the control of domotic systems but could be extended to the monitoring process in industry.

\section{Acknolewgements}

One of the authors is partially suported by $\mathrm{CNPq}$, a Brazilian National Research Agency

\section{References}

Boter, C., Sánchez, L. and Romeral, J. L. (2005). Sistema de control y supervisión remota basada en telefonía móvil gsm.

Chacon, D., Dijort, O. and Castrillo, J. (2001). Supervisión y control de procesos.

Coral, G. M., Rojas, O. A. and Campos, F. A. (2008). Sistema de control y supervisiónn industrial multiplataforma.

Hall, J. (2006). The ibm bisync protocol.

Ramirez, E. Y. R. and Chingate, F. D. (2010). Evaluación, diseño e implementación de un sistema de comunicación remota que permira la adquisición, procesamiento y monitoreo de los datos de una máquina sopladora de botellas en iberplast s.a. 